\title{
BRIÓFITOS COMO BIOINDICADORES DE CALIDAD BOTÁNICA EN ZONAS ÁRIDAS DEL SUDESTE ESPAÑOL: SIERRAS DE FILABRES, CABRERA, ALHAMILLA Y CABO DE GATA (ALMERÍA, ESPAÑA)
}

\author{
Pedro GARCÍA-ZAMORA, Rosa M. ROS y Juan GUERRA
}

RESUMEN. Briófitos como bioindicadores de calidad botánica en zonas áridas del Sudeste español: Sierras de Filabres, Cabrera, Alhamilla y Cabo de Gata (Almería, España). Basado en el estudio de la flora y vegetación briofíticas de las sierras de Filabres, Cabrera, Alhamilla y Cabo de Gata, se ha realizado la evaluación fitobiológica de las mismas aplicando un coeficiente de calidad botánica a una serie de localidades elegidas en el seno de estos sistemas montañosos. De este modo se han cartografiado las zonas con diferentes grados de conservación de la flora y vegetación briofítica en el área de estudio.

Palabras clave. Briófitos, evaluación fitobiológica, coeficiente de calidad botánica, conservación, Filabres, Cabrera, Alhamilla y Cabo de Gata, Almería, SE de España.

SUMMARY. Bryophytes as bioindicators of botanical quality in arid areas of Southeast Spain: Sierras de Filabres, Cabrera, Alhamilla and Cabo de Gata (Almería, Spain). Based in the study of the bryophytic flora and vegetation of the sierras de Filabres, Cabrera, Alhamilla and Cabo de Gata, the phytobiological evaluation of these sierras has been done through the application of a coefficient of botanical quality. To this extent, the zones with different degrees of conservation of the bryophytic flora and vegetation have been mapped.

Key words. Bryophytes, phytobiological evaluation, coefficient of botanical quality, conservation, Filabres, Cabrera, Alhamilla and Cabo de Gata, Almería, SE of Spain.

\section{INTRODUCCIÓN}

Los briófitos son organismos muy sensibles a los factores que impactan negativamente sobre el medio natural como talas, incendios, erosión, presión antropozoógena, etc., por ello la evaluación de la calidad botánica de las sierras estudiadas, utilizándolos como bioindicadores, permite conocer el grado de calidad del territorio desde el punto de vista botánico, lo que es fiel reflejo de su grado de conservación natural. Con estas premisas se han cartografiado las áreas que poseen calidad botánica diferenciada, es decir, los diferentes grados de conservación de la vegetación natural que se presentan en la zona

Trabajo realizado con cargo a los proyectos PB98-111-C01 y HA1997-0138 financiados por la DGES del MEC. 
de estudio. El único antecedente conocido de este método, que tiene en cuenta a los briófitos como indicadores es el de Guerra et al. (1989).

Se han estudiado las sierras de Filabres, Cabrera, Alhamilla y Cabo de Gata, situadas en la provincia de Almería (fig. 1). El conjunto limita al N con el río Almanzora y al NO con la sierra de Baza, continuación de Filabres, marcando el límite el valle del río de las Herrerías. El mar Mediterráneo limita la zona por el E, NE, S y SE. Por el O se encuentra el valle del río Nacimiento y al SO la vertiente oriental de la sierra de Gádor y el río Andarax.

En la sierra de Filabres aparecen principalmente micasquistos grafíticos, cuarcitas, micacitas, anfibolitas, mármoles y gneises y en Alhamilla y Cabrera micasquistos, cuarcitas y filitas con intercalaciones de calizas y/o dolomías. Estos sustratos, con la excepción de los micasquistos que se desagregan fácilmente, permiten el asentamiento de comunidades briofíticas, en unos casos basófilas y en otros acidófilas. La base litológica de la sierra del Cabo de Gata está formada por traquitas y andesitas, rocas que no permiten por su dureza un fácil asentamiento de comunidades briofíticas. El clima presenta una acusada falta de precipitaciones. La precipitación media anual es de $355 \mathrm{~mm}$, siendo siempre superior a los $200 \mathrm{~mm}$, salvo en puntos excepcionales como Cabo de Gata y bajo Almanzora. El régimen térmico es muy suave, con heladas muy raras y restringidas a las zonas más continentales del interior y de alta montaña. La temperatura media anual muestra valores uniformes y altos en el litoral y cuencas bajas de los ríos Almanzora y Almería, oscilando entre $17^{\circ} \mathrm{C}$ y $21^{\circ} \mathrm{C}$, valor que disminuye lenta pero progresivamente al remontar los valles de los ríos, al subir en altura y con la continentalidad. Se trata del territorio más árido y seco de la península Ibérica, de tal forma que las condiciones de sequedad de su litoral y casi la totalidad de su mitad oriental son comparables a las de zonas de Africa del Norte o Próximo Oriente.

\section{METODOLOGÍA}

Para este estudio se han utilizado 115 localidades (fig. 1, anexo 2) que recogen la totalidad de los diferentes tipos de vegetación briofítica y variaciones florísticas que se presentan en el área.

Las localidades se han seleccionado teniendo en cuenta estudios de campo, fotointerpretación y revisión cartográfica. Han sido de gran ayuda los mapas de suelos del proyecto LUCDEME de Aguilar et al. (1987a y b, 1988, 1989 y 1990), Delgado et al. (1991), Ortega et al. (1986) y Pérez-Pujalte et al. (1987, 1989a y b). La intensidad de prospección de cada localidad ha quedado delimitada por la propia experiencia de campo, determinándose así en qué momento se reduce al mínimo la posibilidad de que aparezcan nuevas especies y nichos colonizados por briófitos.

Una vez conocida la brioflora de las localidades y la variabilidad de biótopos ocupados por ellos se ha valorado la calidad botánica de las mismas mediante la aplicación del Índice de Calidad Botánica (Ccb) a cada uno de los sistemas montañosos objeto de este estudio (Géhu \& Géhu, 1980; Guerra et al., 1989):

$$
\mathrm{Ccb}=\mathrm{Cdfl}+\mathrm{Coel}+\mathrm{Nb}
$$

Donde Cdfl es el Coeficiente de Diversidad Florística de una localidad

$$
\mathrm{Cdfl}=\frac{\mathrm{N}^{\circ} \text { de especies presentes en la localidad }}{\mathrm{N}^{\circ} \text { total de especies del área estudiada }} \times 100
$$

y Coel es el Coeficiente de Originalidad Específica de una localidad:

$$
\text { Coel }=\frac{\text { Suma de los Crsp }}{N^{\circ} \text { de especies de la localidad }}
$$




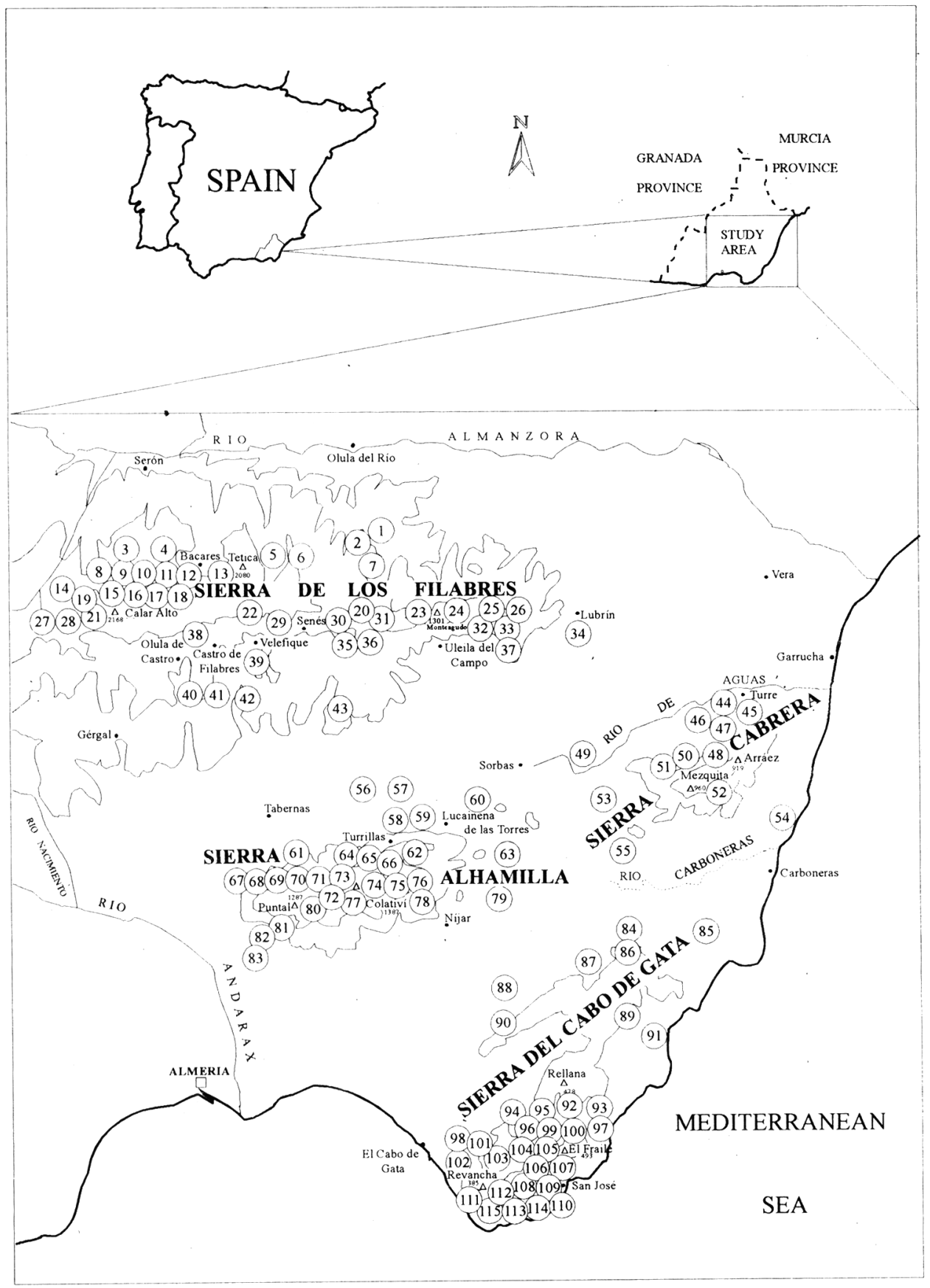

Figura 1. Situación geográfica del área de estudio y localidades estudiadas. Geographic location and studied sites. 
donde Crsp es el Coeficiente de Rareza de una Especie:

$$
\operatorname{Crsp}=\frac{\mathrm{N}^{\circ} \text { total de localidades }-\mathrm{N}^{\circ} \text { de localidades donde está presente }}{\mathrm{N}^{\circ} \text { total de localidades }} \times 100
$$

y $\mathbf{N b}$ es el número de biótopos ocupados por los briófitos.

Las situaciones que se pueden presentar cuando se evalúa cada sistema montañoso o en general, cualquier área, son:

A. Que las áreas sean sensiblemente homogéneas y puedan caracterizarse mediante el estudio de una sola localidad representativa del conjunto.

B. Que las áreas no sean homogéneas y deban ser caracterizadas mediante el estudio de dos o más localidades.

B.1. Cuando el área no es muy extensa, todas las localidades se prospectan con la misma intensidad. En este caso, se utiliza el valor medio del coeficiente de calidad botánica (Ccb) de todas ellas.

B.2. Para las áreas muy extensas y con el fin de completar su estudio y obtener datos fiables, unas localidades se estudian en profundidad y otras de forma más somera. Con los datos de las localidades estudiadas menos profundamente, se calcula un solo valor de $\mathrm{Ccb}$, que posteriormente se integrará con el resto, para calcular un valor medio.

Basándose en los datos obtenidos, se han diferenciado, en cada sierra estudiada, 4 grados de calidad botánica, marcados por los siguientes rangos: áreas de calidad botánica 1 (Ccb de 65 a 80), 2 (Ccb de 80 a 86), 3 (Ccb de 86 a 96) y 4 (Ccb de 96 a 125). La determinación de estos rangos se ha hecho valorando en conjunto los datos de Ccb de todas las localidades estudiadas y aspectos relativos a la vegetación real cormofítica existente en cada una de ellas. En general, un buen estado de conservación de la vegetación cormofítica suele implicar una vegetación briofítica en consonancia.

Todos los datos de flora y vegetación briofíticas se basan en los trabajos Cano et al. (1999), García-Zamora et al. (1998), GarcíaZamora et al. (en prensa). Nomenclatura de táxones y sintáxones sigue respectivamente a Casas (1991) y Marstaller (1993). Anexo 1.

\section{RESULTADOS}

\section{A. SIERRA DE LOS FILABRES}

\section{Áreas de calidad botánica 1}

Comprenden dos pequeñas zonas en el norte de Filabres, una en la parte noroccidental y otra en la nororiental. En sus vertientes sur y suroriental forman una franja que llega hasta la localidad de Tabernas, formando un pasillo en dirección sudeste que alcanza Cabrera (fig. 2). La evaluación fitobiológica se basa en el estudio de las localidades $8,20,24,25,29,32,39$ y 42 . Han sido caracterizadas por los índices de calidad botánica comprendidos entre 67.6 y 79.1, dando un Ccb medio de 74.4. Los valores de Cdfl, Coel y Nb medios para las localidades de estas áreas (tab. 1) implican un pequeño número de especies, muy comunes, que ocupan un reducido número de hábitats diferentes (como máximo 3). La vegetación cormofítica que aparece en estas zonas de calidad botánica 1 es principalmente de la serie Adenocarpo decorticantis-Querceto rotundifoliae $S$. en su faciación mesomediterránea de Retama sphaerocarpa, en cotas entre los 1000 y 1400 $\mathrm{m}$, dentro del termótipo mesomediterráneo y bajo ombrótipo seco. Los briófitos de la zona soportan fuertes exposiciones y colonizan rocas o suelos secos, arenosos, pedregosos y sueltos o bien protosuelos, por lo que las comunidades de estas zonas de calidad botánica 1 son: Brachythecietum albicantis (CeratodontoPolytrichetea piliferi); Reboulio hemisphaericaeTargionietum hypophyllae, Homalothecio aureiPleurochaetetum squarrosae, Crossidio 

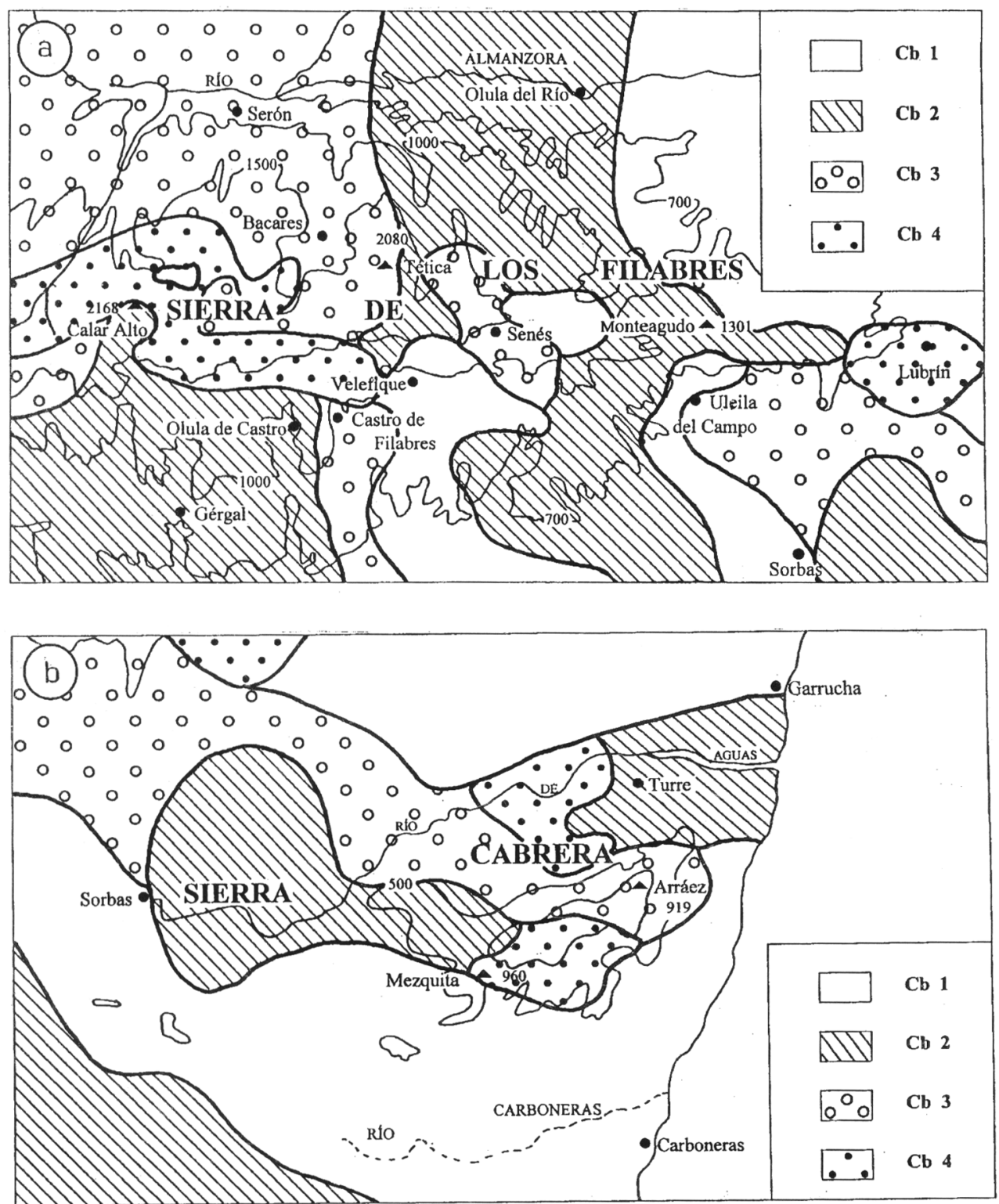

$4 \mathrm{~km}$

Figura 2. Áreas de calidad botánica de la sierra de los Filabres (a) y áreas de calidad botánica de la sierra de Cabrera (b). Areas of botanical quality in Sierra de Filabres (a) and areas of botanical quality in Sierra de Cabrera (b). 
squamiferi-Aloinetum aloidis (Barbuletea unguiculatae) y una comunidad de Grimmia pulvinata (Grimmietea anodontis). En su conjunto son comunidades frecuentes en todo el SE español, sobre todo aquellas que se desarrollan sobre sustratos básicos.

\section{Áreas de calidad botánica 2}

Ocupan dos amplias zonas en Filabres, una en la parte suroccidental que discurre por su vertiente meridional, siguiendo la dirección de los ríos Nacimiento y Andarax, hasta casi su desembocadura en el golfo de Almería y otra en la nororiental, desde los alrededores de Senés hasta Lucainena de las Torres, ya en Alhamilla. Aparece además una pequeña mancha en los alrededores del pico Tetica de Bacares y otra en las cercanías del Calar Alto (fig. 2). El estudio y la evaluación fitobiológica se realizó con las localidades 1, 2, 5, 7, 19, 22, $23,27,31,33,36,40$ y 43 , que muestran un Ccb medio de 82.1. Los Cdfl, Coel y Nb medios (tab. 1) difieren de los de las áreas de calidad botánica 1, sobre todo en la diversidad florística, lo que implica un mayor número de especies por localidad, aunque siguen siendo táxones muy comunes y ocupan un reducido número de hábitats, que pueden llegar en algunos casos a 4. Los afloramientos rocosos son muy abundantes donde la pendiente es más fuerte, quedando suelo en las grietas de las rocas y en vaguadas, lo que determina la presencia de comunidades briofíticas no muy frecuentes, propias de estos hábitats, como: Hedwigio-Orthotrichetum rupestris (Racomitrietea heterostichi), Homalothecio sericei-Leptodontetum smithii (Neckeretea complanatae). También aparecen comunidades más comunes pertenecientes a la clase Barbuletea unguiculatae (Crossidio squamiferi-Aloinetum aloidis, Homalothecio aurei-Pleurochaetetum squarrosae, Homalothecio aurei-Scleropodietum touretii y Acaulo triquetri-Tortuletum brevissimae), aunque quedan grandes áreas en las que no hay ninguna comunidad bien estructurada (un 28\% de las localidades estudiadas). La vegetación cormofítica varía, según la altitud, desde un matorral subserial-serial ligeramente aclarado de Adenocarpo decorticantis-Querceto rotundifoliae $S$. o retamares de Rhamno lycioidis-Querceto cocciferae $S$. en el termótipo mesomediterráneo, que tienen mayor cobertura en las cotas menores, hasta un piornal en cotas cercanas a los $1700 \mathrm{~m}$. En el termótipo supramediterráneo se puede encontrar, muy puntualmente, la serie Daphno oleoidi-Pineto sylvestris $S$. formada por un pinar abierto, con un sotobosque de enebros y sabinas con muchas espinosas pero muy degradado.

\section{Áreas de calidad botánica 3}

Ocupan una amplia zona en la parte septentrional de la vertiente norte, que va desde las partes más altas de la sierra $(2000 \mathrm{~m})$ hasta cotas más bajas $(800 \mathrm{~m})$. Aparecen cuatroáreas más, una en la parte occidental (localidad 26), otra en la oriental (localidades 21, 27 y 28), la tercera que va por la ladera sur de la sierra, desde Castro de Filabres hacia Tabernas y otra que ocupa los alrededores de Senés y se prolonga hacia el norte (localidades 6, 30 y 35 ) (fig. 2). Se caracterizan por los índices de calidad botánica entre 86.7 y 94.6, con un Ccb medio de 90.3. El estudio y la evaluación fitobiológica se realizó con las localidades 4 , $6,10,13,15,16,18,26,30,35,37$ y 41 de esta sierra. Los Cdfl, Coel y Nb medios (tab. 1) difieren de los obtenidos en las áreas de calidad botánica 1 y 2 , principalmente en el de diversidad florística, ya que hay más especies en cada localidad y van apareciendo algunas especies menos comunes. Los hábitats que ocupan los briófitos siguen siendo semejantes a los del área de calidad botánica 2, aunque pueden colonizar 3 ó 4 biótopos en cada localidad. Se han detectado las asociaciones Eucladio verticillati-Didymodontetum tophacei (clase Montio-Cardaminetea); HedwigioOrthotrichetum rupestris (clase Racomitrietea 
heterostichi), Gymnostomo luisieriSouthbyetum nigrellae, Reboulio hemisphericae-Targionietum hypophyllae, Homalothecio aurei-Pleurochaetetum squarrosae, Homalothecio aureiScleropodietum touretii, Tortulo subulataeSyntrichietum ruralis, Acaulo triquetriTortuletum brevissimae, Funarietum hygrometricae y Crossidio squamiferiAloinetum aloidis (clase Barbuletea unguiculatae); comunidades de Grimmia pulvinata (clase Grimmietea anodontis) y la asociación Homalothecio sericeiLeptodontetum smithii (clase Neckeretea complanatae), quedando escasas áreas (17\%) sin comunidades briofíticas bien definidas. Aunque ninguna de estas comunidades puede considerarse rara, en conjunto se manifiesta una elevada diversidad fitocenótica.

\section{Áreas de calidad botánica 4}

Están representadas por pequeñas zonas, muy localizadas y protegidas, donde se conservan densos núcleos de vegetación natural. Aparece en una amplia franja al sur y sudoeste que va desde Castro de Filabres hasta el Calar Alto ocupando las zonas más altas en cotas superiores a los $2000 \mathrm{~m}$ y se prolonga en la vertiente norte bajando hasta cotas de 1500 m aproximadamente, donde existen un número importante de barrancos que recogen el agua de deshielo en primavera. En la zona más oriental aparece otra pequeña área en los alrededores de Lubrín (fig. 2). Han sido caracterizadas por los índices de calidad botánica comprendidos entre los valores 94.6 y 123.4, con un Ccb medio de 105.4 y la evaluación fitobiológica se ha realizado con el estudio de las localidades $3,9,11,12,14,15$, 17, 21, 28, 34 y 38. El Cdfl es muy alto debido a la presencia de especies como Philonotis fontana y Calliergonella cuspidata, con unos Crsp de 96.5 y de 97.4, respectivamente, lo que determina un aumento drástico del Coel y consiguientemente del Ccb. Destaca el elevado $\mathrm{Nb}$ que presenta la mayoría de localidades (excepto la 28) que oscila entre 4 y 6 , debido principalmente a la presencia de una vegetación cormofítica natural de Adenocarpo decorticantis-Querceto rotundifoliae $S$. formada por bosquetes de Quercus rotundifolia, Adenocarpus decorticans, Juniperus oxycedrus. En algunas zonas altas $(1800 \mathrm{~m})$ aparece la serie Genisto baeticae-Junipereto nanae $S$. Muy puntualmente hay un coscojar denso y elevado de la serie Rhamno lycioidisQuerceto cocciferae $S$. Se han encontrado comunidades de todas las clases de vegetación briofítica detectadas en la zona de estudio. De Montio-Cardaminetea (Eucladio verticillatiDidymodontetum tophacei y Rhizomnio punctati-Fissidentetum taxifolii y la comunidad de Preisia quadrata), de PlatyhypnidioFontinalietea antipyreticae (Oxyrrhynchietum rusciformis); de Ceratodonto-Polytrichetea piliferi (Brachythecietum albicantis); de Racomitrietea heterostichi (HedwigioOrthotrichetum rupestris); de Dicranelletea heteromallae (Bartramietum pomiformis y las comunidades de Bartramia ithyphylla y Amphidium mougeotii); de Barbuletea unguiculatae (Reboulio hemisphaericaeTargionietum hypophyllae, Homalothecio aurei-Scleropodietum touretii y Crossidio squamiferi-Aloinetum aloidis); de Neckeretea complanatae (Homalothecio sericeiLeptodontetum smithii) y de FrullanioLeucodontetea sciuroidis (Syntrichietum laevipilae). Destaca la presencia de raras especies como Acaulon fontiquerianum, Amphidium mougeotii, Atrichum undulatum, Brachythecium dieckii, Cephaloziella stellulifera, Homalothecium philippeanum, Orthotrichum macrocephalum, Scapania praetervisa, Schistidium pulchrum y Schistidium singarense, todas ellas con elevados Crsp que confieren al área este alto grado de calidad botánica. 


\section{B. SIERRA DE CABRERA}

\section{Áreas de calidad botánica 1}

Ocupan gran parte de las vertientes norte, sur y este de la sierra, prolongándose a lo largo de la costa hasta la sierra del Cabo de Gata (fig. 2), siempre dentro del termótipo termomediterráneo bajo ombrótipo semiárido y en cotas que oscilan de los 0 hasta los $300 \mathrm{~m}$ aproximadamente. La evaluación fitobiológica se ha realizado con las localidades 53,54 y 55 , caracterizadas por los índices de calidad botánica comprendidos entre 76 y 79.1, dando como resultado un $\mathrm{Ccb}$ medio para estas áreas de 76.1. Los Cdfl, Coel y Nb medios para las localidades de estas áreas (tab. 1) implican un reducido número de especies, muy comunes y que ocupan un pequeño número de hábitats (como máximo 2). Los suelos predominantes se desarrollan en pendientes suaves, poco pedregosas, con poca materia orgánica y casi siempre con un déficit de agua importante, lo que hace que estén secos grandes periodos. Esto provoca en muchas áreas la aparición de bad-lands y que la vegetación se reduzca a un matorral-pastizal xérico de escasa cobertura (del 20 al $50 \%$ ) de las series Chamaeropo humilis-Rhamneto lycioidis S. y Mayteneto europaei-Periploceto angustifoliae S. Los briófitos de la zona disponen, fundamentalmente, de suelos poco pedregosos o protosuelos y por ello las comunidades briofíticas que aparecen en estas zonas de calidad botánica 1 son de la clase Barbuletea unguiculatae (Trichostomo crispuli-Aloinetum aloidis, Selaginello denticulatae-Timmielletum barbuloidis, Crossidio crassinervis-Tortuletum obtusatae y Crossidio crassinervis-Aloinetum aloidis).

\section{Áreas de calidad botánica 2}

Aparecen dos zonas, una desde las cotas cercanas a los $700 \mathrm{~m}$ de altitud, en la cara suroccidental de la sierra hasta Sorbas y otra en el norte de la sierra en los alrededores de
Turre y Garrucha que llega hasta la costa (fig 2), siempre dentro del termótipo termomediterráneo, bajo ombrótipo semiárido y en cotas que oscilan de los 0 a los $550 \mathrm{~m}$. El estudio y la evaluación fitobiológica se ha realizado con las localidades $45,46,49$ y 51 , caracterizadas por los índices de calidad botánica comprendidos entre 79.6 y 85.3 , que dan un Ccb medio de 83.4. Los Cdfl, Coel y Nb medios (tab. 1) son ligeramente diferentes a los obtenidos en las áreas de calidad botánica 1, sobre todo en los correspondientes a la diversidad florística, lo que implica, al igual que en Filabres, un mayor número de especies por localidad, aunque en general siguen siendo bastante comunes y ocupando un reducido número de hábitats (como máximo 3). Los suelos se desarrollan en pendientes medias, con elevado contenido en arena y arcilla, poco profundos y con una baja retención de agua, sufriendo una intensa erosión favorecida por la escasa cobertura vegetal de pastizal matorra de la serie Zizipheto loti $S$. en las cotas más bajas (170-180 m) o espartales de Chamaeropo humilis-Rhamneto lycioidis $S$. en cotas algo superiores $(400-550 \mathrm{~m})$. Los briófitos de la zona disponen de suelos poco pedregosos 0 protosuelos y por ello las comunidades briofíticas que aparecen son principalmente de la clase Barbuletea unguiculatae (Gymnostomo luisieri-Southbyetum nigrellae, Selaginello denticulatae-Timmielletum barbuloidis, Crossidio crassinervis-Aloinetum aloidis y Crossidio crassinervis-Pottietum commutatae) y más raramente de Grimmietea anodontis (Grimmietum orbicularis).

\section{Áreas de calidad botánica 3}

Ocupan parte de la vertiente occidental formando una franja que llega hasta el pico Arráez y sus alrededores (fig. 2). Están caracterizadas por los índices de calidad botánica comprendidos entre 86.1 y 90.5 , con un Ccb medio de 88.3. La evaluación fitobiológica se realizó con las localidades 48 

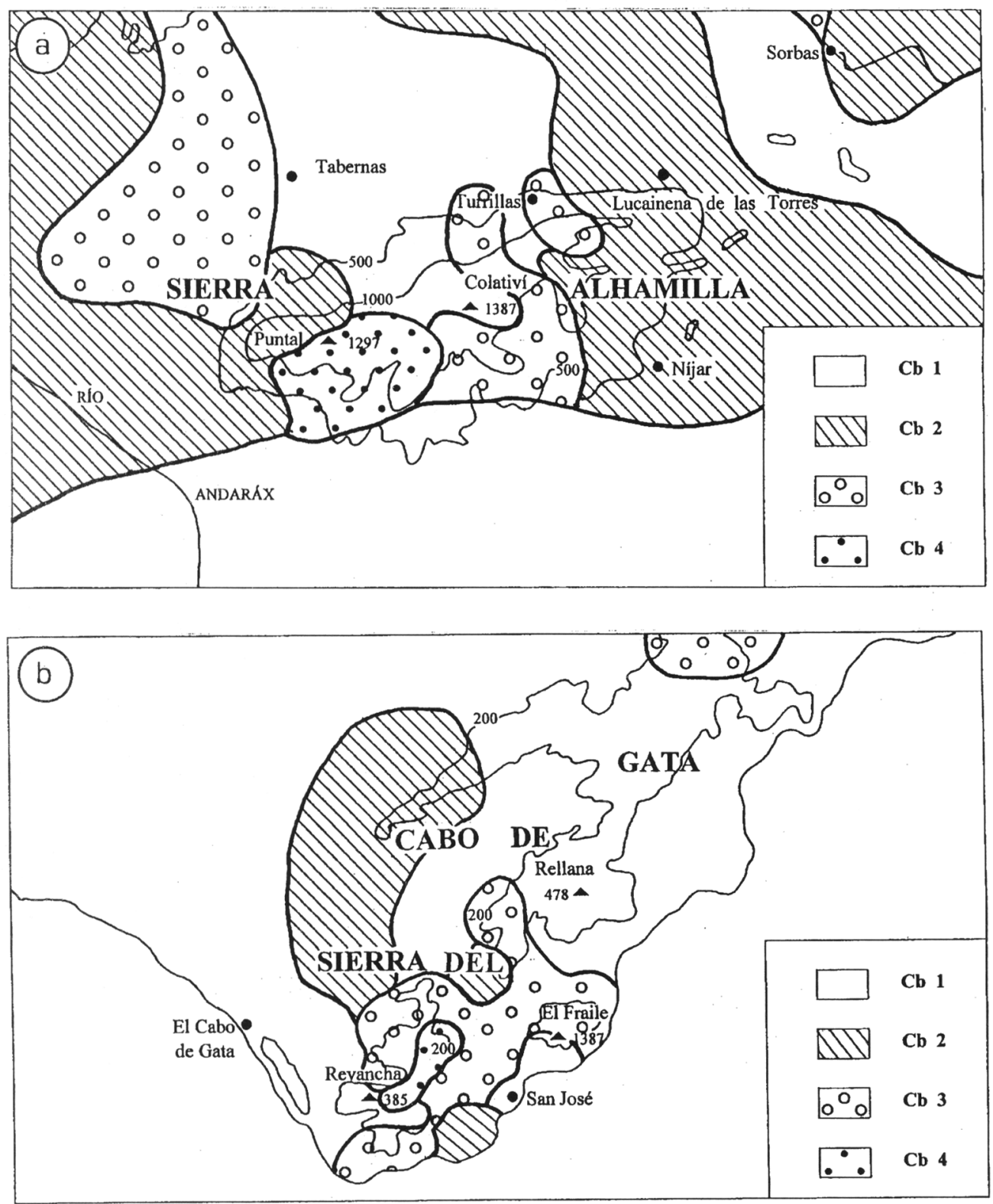

$4 \mathrm{~km}$

Figura 3. Áreas de calidad botánica de la sierra de Alhamilla (a) y áreas de calidad botánica de la sierra del Cabo de Gata (b). Areas of botanical quality in Sierra de Alhamilla (a) and areas of botanical quality in Sierra del Cabo de Bata (b). 
y 50. Los Cdfl, Coel y Nb medios (tab. 1) difieren de los que se han obtenido en las áreas de calidad botánica 2, sobre todo en los correspondientes a la diversidad florística, pues los coeficientes de originalidad específica y los $\mathrm{Nb}$ son prácticamente iguales, lo que implica un mayor número de especies por localidad, aunque siguen siendo bastante comunes y ocupando un pequeño número de hábitats. Abundan suelos en pendientes medias, poco profundos y con una baja retención de agua útil, con una erosión importante favorecida por la escasa cobertura vegetal de pastizal matorral (20-25\%) de la serie de vegetación Chamaeropo humilis-Rhamneto lycioidis S., que en condiciones favorables (umbrías por lo general) forma un bosquete muy denso con lentiscos, palmitos, espinos, etc. En otras zonas aparece la serie Smilaci mauritanicae-Querceto rotundifoliae $S$., que forma un bosque denso de encinas. Sólo en pequeñas capas de tierra o protosuelos se asientan muchas especies de briófitos de las asociaciones Gymnostomo luisieri-Southbyetum nigrellae, Selaginello denticulatae-Timmielletum barbuloidis y Crossidio crassinervis-Aloinetum aloidis, todas ellas de la clase Barbuletea unguiculatae.

\section{Áreas de calidad botánica 4}

Al igual que en Filabres están representadas por pequeñas manchas, bastante localizadas y protegidas, donde se conservan densos núcleos de vegetación natural. Sierra Cabrera presenta dos áreas que se sitúan a ambos lados del pico Arráez, de 919 m, una en la vertiente sur de la sierra (localidad 52) y otra en la norte (localidades 44 y 47), situadas entre los 180 y los $850 \mathrm{~m}$ (fig. 2) dentro de los termótipos termo- y mesomediterráneo bajo ombrótipo seco o semiárido. Han sido caracterizadas por los índices de calidad botánica comprendidos entre 96.9 y 104.9 , con un Ccb medio de 102.2. La evaluación fitobiológica se ha realizado con el estudio de las localidades 44, 47 y 52. Los Cdfl, Coel y
Nb medios (tab. 1), al igual que en Filabres, se separan de forma importante de los obtenidos en áreas de calidad botánica inferior, debido, sobre todo, a que aparece un número importante de biótopos donde los briófitos encuentran el medio adecuado para su desarrollo (hasta 7 diferentes). Abundan suelos formados en pendientes suaves por lo que son profundos y poco pedregosos aunque con una baja retención de agua. En las zonas más bajas de estas áreas aparecen espesos matorrales de azufaifos de la serie de vegetación Zizipheto loti $S$, formándose bajo ellos un microhábitat donde se desarrollan muchos nanofanerófitos y caméfitos y diversas comunidades briofíticas, principalmente de la clase Barbuletea unguiculatae (Gymnostomo luisieriSouthbyetum nigrellae, Riccio nigrellaeOxymitretum paleaceae, Plagiochasmo rupestris-Athalamietum spathysii especialmente rara en el sudeste español Selaginello denticulatae-Timmielletum barbuloidis, Acaulo triquetri-Tortuletum brevissimae, Crossidio crassinervis-Aloinetum aloidis). Puntualmente aparece la comunidad de Grimmia pulvinata, de la clase Grimmietea anodontis. Las especies que aparecen en estas áreas y que contribuyen con su coeficiente de rareza a que posean este grado de alta calidad botánica son, entre otras: Athalamia spathysii, Crossidium aberrans, Didymodon sicculus, Phascum cuynetii, Pterygoneurum sampaianum.

\section{SIERRA DE ALHAMILLA}

\section{Áreas de calidad botánica 1}

Se presentan en la falda norte de Alhamilla, en altitudes comprendidas entre 1100 y $1350 \mathrm{~m}$ y en casi toda su vertiente meridional en cotas menores de $700 \mathrm{~m}$ (fig. 3). El estudio y la evaluación fitobiológica se realizaron con las localidades 56, 60, 61, 65, $70,71,72,73,74$ y 83 y se han caracterizado por los índices de calidad botánica 
comprendidos entre 71.8 y 79.1 , con un $\mathrm{Ccb}$ medio de 77.7. Los Cdfl, Coel y Nb medios (tab. 1) implican un pequeño número de especies, muy comunes y que ocupan un reducido número de hábitats, al igual que en el resto de las sierras. La vegetación cormofítica es principalmente de la serie Adenocarpo decorticantis-Querceto rotundifoliae $S$. en su faciación mesomediterránea de Retama sphaerocarpa que se sitúa en cotas entre los 1200 y 1350 m, bajo ombrótipo seco. En cotas menores (200-700 m) pueden aparecer las series Rhamno lycioidis-Querceto cocciferae S., Paeonio coriaceae-Querceto rotundifoliae $S$. y también la serie Zizipheto loti S. En estas condiciones la clase de vegetación briofítica dominante es Barbuletea unguiculatae (Homalothecio aurei-Pleurochaetetum squarrosae, Trichostomo crispuli-Aloinetum aloidis, Crossidio crassinervis-Aloinetum aloidis y Crossidio squamiferi-Aloinetum aloidis). También aparecen algunos epífitos, representados, muy puntualmente, por la asociación Syntrichietum laevipilae, de la clase Frullanio-Leucodontetea sciuroidis.

\section{Áreas de calidad botánica 2}

Ocupa las estribaciones oriental y occidental, por donde se prolonga hacia la costa y alcanza la sierra del Cabo de Gata en su vertiente nororiental (fig. 3). El estudio y la evaluación fitobiológica se realizó con las localidades 57, 58, 59, 63, 66, 68, 69, 78, 79 y 82, de Ccb medio 82.5. Los Cdfl, Coel y $\mathrm{Nb}$ medios (tab. 1) difieren de los obtenidos en las áreas de calidad botánica 1, sobre todo en los correspondientes a la diversidad florística, lo que implica un mayor número de especies por localidad aunque en general siguen siendo táxones bastante comunes. La vegetación natural está formada por un pastizal-matorral, principalmente de las series Rhamno lycioidisQuerceto cocciferae S. y Zizipheto loti S., aunque también hay zonas donde aparecen las series Rhamno lycioidis-Querceto cocciferae
S. y Paeonio coriaceae-Querceto rotundifoliae $S$. como en la zonas de calidad botánica 1 . Generalmente suele aparecer una sola comunidad briofítica en cada localidad, normalmente de la clase Barbuletea unguiculatae. Sólo en una ocasión se ha encontrado la asociación Brachythecietum albicantis (clase Ceratodonto-Polytrichetea piliferi) y en otra, la asociación Syntrichietum laevipilae (clase Frullanio-Leucodontetea sciuroidis). Hay también zonas en las que no hay ninguna comunidad bien estructurada (hasta un 20\% de las localidades prospectadas).

\section{Áreas de calidad botánica 3}

Estas áreas van desde Tabernas hasta el pico del Puntal. Además, alrededor del pico Colativí se encuentra una zona de calidad botánica 3, situada en cotas por encima de los $500 \mathrm{~m}$, que se prolonga hacia ambas vertientes de la sierra y una pequeña mancha en Turrillas (fig. 3). Caracterizadas por los índices de calidad botánica comprendidos entre 87.1 y 93.6, con un Ccb medio de 90.6. El estudio y la evaluación fitobiológica se realizó con las localidades 62, 64, 67, 75, 76 y 77. Los Cdfl y Coel medios (tab. 1) son semejantes a los de las otras sierras estudiadas, sin embargo aumenta ligeramente el número de biótopos colonizados por briófitos (en estas áreas es generalmente de 4 por localidad) y aparecen diversas especies infrecuentes en todo el territorio. Las zonas más bajas están ocupadas por matorrales de la serie Zizipheto loti S. y en cotas mayores se mantienen formaciones de tomillares, espartales, romerales, albaidares y más raramente retamares, lentiscares o coscojares. También aparecen restos de un encinar, de particular interés. Se han detectado varias clases de vegetación briofítica, a destacar: Barbuletea unguiculatae (Homalothecio aureiPleurochaetetum squarrosae, Homalothecio aurei-Scleropodietum touretii), CeratodontoPolytrichetea piliferi (comunidad de Anacolia menziesii), Frullanio-Leucodontetea sciuroidis 
(Syntrichietum laevipilae) y Racomitrietea heterostichi (Hedwigio-Orthotrichetum rupestris). Es destacable que el Cdfl es mayor incluso que el de las áreas de calidad botánica 4 , y que el Coel sin embargo se diferencia poco del de las áreas de calidad botánica 2. Las únicas especies que aparecen en estas áreas y que presentan un Coel elevado son principalmente Anacolia menziesii, Orthotrichum acuminatum, $O$. pumilum, $O$. tenellum, $O$. tortidontium y $O$. urnigerum.

\section{Áreas de calidad botánica 4}

Sólo aparece una pequeña zona en los alrededores del pico Puntal $(1287 \mathrm{~m})$ que se prolonga en la cara sur de la sierra desde los $1200 \mathrm{~m}$ y alcanza cotas cercanas a $\operatorname{los} 400 \mathrm{~m}$ (fig. 3). Han sido caracterizadas por los índices de calidad botánica comprendidos entre 96.2 y 100.3, con un Ccb medio de 98.3. La evaluación fitobiológica se ha realizado con el estudio de las localidades 80 y 81 . Los Cdfl, Coel y Nb medios (tab. 1) difieren de manera muy notable de los obtenidos en las dos sierras anteriores para este mismo grado de calidad botánica. En este caso el Coel destaca sobre los otros coeficientes, debido principalmente a la presencia de especies con elevados Crsp, que aumentan drásticamente el Cdfl y por tanto el Ccb, entre ellas Fabronia pusilla, Orthotrichum macrocephalum, O. pumilum y Tortula ruraliformis var. subpapillosissima. Las comunidades briofíticas que aparecen son Hedwigio-Orthotrichetum rupestris (clase Racomitrietea heterostichi) Homalothecio sericei-Leptodontetum smithii (clase Neckeretea complanatae) y Syntrichietum laevipilae (clase Frullanio-Leucodontetea sciuroidis). Estas zonas están más protegidas de la fuerte sequía circundante y en ellas se conservan núcleos más densos de vegetación natural formada por espesos matorrales de azufaifos de la serie Ziziphetum loti S., bajo los cuales pueden aparecer comunidades briofíticas. También se puede encontrar la etapa madura de la serie Paeonio coriaceae-Querceto rotundifoliae $S$., con un bosque de gran talla de Quercus rotundifolia y un matorral denso, medio idóneo para el asentamiento de las comunidades epífitas. No obstante el $25 \%$ de las localidades prospectadas no presenta comunidades bien estructuradas.

\section{SIERRA DEL CABO DE GATA}

\section{Áreas de calidad botánica 1}

Desde Alhamilla estas áreas se prolongan hacia el este ocupando casi la totalidad de La Serreta de Níjar, gran parte del norte de la sierra del Cabo de Gata y las zonas costeras de las salinas de Acosta y del pueblo de San José (fig. 3). El estudio y la evaluación fitobiológica se realizó con las localidades 85, 87, 88, 89, 91, 92, 93, 94, 97, 102, 105, 109, 111, 112 y 113, caracterizadas por los índices de calidad botánica comprendidos entre 68.7 y 78.9 , dando como resultado un $\mathrm{Ccb}$ medio de 75.6. Los Cdfl, Coel y Nb medios (tab. 1), implican un escaso número de especies, en general muy comunes y que ocupan un reducido número de biótopos (1 en todas las localidades). Las localidades (salvo una) se encuentran en el termótipo termomediterráneo con ombrótipo semiárido, en altitudes comprendidas entre los 0 y $\operatorname{los} 400 \mathrm{~m}$. La vegetación cormofítica que aparece en estas zonas es principalmente de las series Mayteneto europaei-Periploceto angustifoliae S. y Zizipheto loti S. En ambos casos se trata de una vegetación de escasa cobertura representada por tomillares muy aclarados, algún que otro espartal y pastizales de gramíneas con una cobertura del 20-30\% . Las comunidades briofíticas que aparecen son, sobre todo, de la clase Barbuletea unguiculatae (muy frecuentemente Trichostomo crispuliAloinetum aloidis, y más raramente Riccio nigrellae-Oxymitretum paleaceae, FissidentoFunarietum curvisetae, Acaulo triquetriTortuletum brevissimae, Crossidio crassinervis- 
Pottietum commutatae). Es destacable también la presencia de un importante número de localidades $(30 \%)$ que no presentan ninguna comunidad briofítica bien estructurada.

\section{Áreas de calidad botánica 2}

En la vertiente noroccidental de esta sierra se observa un área que ocupa gran parte de los campos de Níjar y dos pequeñas zonas, una en las cercanías de la población de El Pozo de los Frailes y otra en la zona costera del sur de la sierra (fig. 3). En este caso los índices de calidad botánica se hallan comprendidos entre 80.9 y 85.7 , dando como resultado un $\mathrm{Ccb}$ medio para estas áreas de 82.9. El estudio y la evaluación fitobiológica se realizó con las localidades 84, 90, 96, 98, 110 y 114. Los Cdfl, Coel y $\mathrm{Nb}$ medios (tab. 1) integran, como corresponde a este tipo de área, un escaso número de especies, en general muy comunes y que ocupan un reducido número de biótopos distintos (en casi todos los casos 1). Todas las localidades de estas áreas (salvo una) se encuentran en el termótipo termomediterráneo con ombrótipo semiárido en altitudes comprendidas entre $\operatorname{los} 0$ y $\operatorname{los} 250 \mathrm{~m}$. La vegetación cormofítica que aparece es la misma que en las zonas de calidad botánica 1, aunque localmente se encuentra también la serie Chamaeropo humilis-Rhamneto lycioidis S. Los briófitos encontrados en estas zonas son, en todos los casos, de la clase Barbuletea unguiculatae, estando representadas sólo las asociaciones Riccio nigrellae-Oxymitretum paleaceae y Crossidio crassinervis-Pottietum commutatae. Más del $30 \%$ de las localidades no presentan ninguna comunidad briofítica bien estructurada.

\section{Áreas de calidad botánica 3}

Aparecen dos zonas, una pequeña en los límites nororientales de la sierra, en cotas cercanas a los $200 \mathrm{~m}$, y otra que rodea a las zonas culminales y se extiende por la vertiente sureste hasta alcanzar al mar en dos puntos, uno en la localidad 100 (rambla del Pozo de los Frailes) y otro en el faro de Gata (localidad 115) (fig. 3). Están caracterizadas por los índices de calidad botánica comprendidos entre 87.8 y 95.5, con un Ccb medio de 91.9. El estudio y la evaluación fitobiológica se realizó con las localidades 86, 95, 99, 100, 101, 104, 106, 107, 108 y 115. Los Cdfl, Coel y Nb medios son respectivamente $13,76.1$ y 3.2 , los cuales se diferencian principalmente en el Cdfl y $\mathrm{Nb}$, con respecto a los casos anteriores. De hecho, el número de biótopos que ocupan los briófitos, en cada localidad de estas áreas, es generalmente de 3-4, pudiendo llegar hasta 6 . Aparecen varias localidades con especies muy poco comunes, destacan Crossidium seriatum, Funariella curviseta, Gigaspermum mouretti, Gongylanthus ericetorum, Pottia pallida, Pyramidula tetragona, que contribuyen con sus elevados Crsp a que aumente el coeficiente de calidad botánica medio de estas zonas. Todas las localidades están en el termótipo termomediterráneo con ombrótipo semiárido, en altitudes comprendidas entre los 0 y los 400 $\mathrm{m}$. La vegetación cormofítica que aparece es la misma que en las zonas de calidad botánica 1, series Mayteneto europaei-Periploceto angustifoliae S. y Zizipheto loti S. La diversidad florística no tiene reflejo en la fitocenológica, porque la mayoría de las comunidades briofíticas corresponden a la clase Barbuletea unguiculatae, entre ellas Riccio nigrellaeOxymitretum paleaceae, Trichostomo crispuliAloinetum aloidis, etc. y sólo una a Neckeretea complanatae (Homalothecio sericeiLeptodontetum smithii).

\section{Áreas de calidad botánica 4}

La sierra del Cabo de Gata sólo presenta una localidad con un Ccb lo suficientemente alto para ser incluída en este grupo, es la localidad 103, que se sitúa, con una altitud de $180 \mathrm{~m}$, en la vertiente norte del pico Revancha (fig. 3), en valles bastante cerrados y protegidos por la propia sierra de los fuertes vientos 


\begin{tabular}{cccccc}
\hline SIERRA & $\begin{array}{c}\text { Á rea de calidad } \\
\text { botánica }\end{array}$ & $\begin{array}{c}\text { Cdfl } \\
\text { medi }\end{array}$ & $\begin{array}{c}\text { Coel } \\
\text { medio }\end{array}$ & Nb medio & Ccb medio \\
\hline Filabres & $\mathbf{1}$ & 5 & 67.8 & 1.6 & 74.4 \\
& $\mathbf{2}$ & 7 & 73 & 2 & 82.1 \\
& $\mathbf{3}$ & 8.6 & 79.3 & 2.3 & 90.3 \\
Cabrera & $\mathbf{4}$ & 15.2 & 85.7 & 4.2 & 105.4 \\
& $\mathbf{1}$ & 5.4 & 69.4 & 1.3 & 76.1 \\
& $\mathbf{2}$ & 7.3 & 73.9 & 2.3 & 83.4 \\
& $\mathbf{3}$ & 11.3 & 74 & 3 & 88.3 \\
Alhamilla & $\mathbf{4}$ & 18.5 & 78.4 & 5.3 & 102.2 \\
& $\mathbf{1}$ & 4.7 & 72 & 1.6 & 77.7 \\
& $\mathbf{2}$ & 7.2 & 73.7 & 1.6 & 82.5 \\
Cabo de & $\mathbf{3}$ & 9.8 & 77.5 & 3.2 & 90.6 \\
Gata & $\mathbf{4}$ & 3.6 & 92.2 & 2.5 & 98.3 \\
& $\mathbf{1}$ & 4 & 70.6 & 1 & 75.6 \\
& $\mathbf{2}$ & 5.2 & 76.5 & 1.2 & 82.9 \\
& $\mathbf{3}$ & 13 & 76.1 & 3.2 & 91.9 \\
& $\mathbf{4}$ & 19 & 79.6 & 3 & 102.6 \\
\hline
\end{tabular}

Tabla 1. Coeficientes de diversidad florística (Cdfl), de originalidad específica (Coel), número de biótopos (Nb) y coeficiente de calidad botánica (Ccb) para las áreas de calidad botánica de cada sierra estudiada. Coefficients of floristic diversity (Cdfl), specific originality (Coel), biotope number (Nb) and coefficient of botanical quality $(C \mathrm{Cb})$ for the different areas of botanical quality of each of the studied sierras.

procedentes del mar. La vegetación cormofítica en esta localidad pertenece a la serie Zizipheto loti $S$. con una cobertura máxima del $60 \%$. Son destacables el elevado Cdfl (19) y los bajos valores medios del Coel (79.6). Es importante la presencia de especies como Acaulon fontiquerianum, Athalamia spathysii, Didymodon sicculus, Funariella curviseta, Tortella flavovirens. var. papillosissima y Tortula israelis. El número medio de biótopos ocupados por los briófitos (3) comparado con los obtenidos en las áreas de calidad botánica 3 de esta misma sierra, es igualmente bajo para un área de calidad botánica 4. Esto implica que el alto Ccb obtenido (102.6) se debe primordialmente a una importante diversidad específica, ya que las comunidades briofíticas son escasas y reducidas a pocos casos de la clase Barbuletea unguiculatae (Fissidento-
Funarietum curvisetae y Acaulo triquetriTortuletum brevissimae) y más raramente de la clase Grimmietea anodontis (comunidad de Grimmia pulvinata).

\section{CONCLUSIONES}

Las áreas de calidad botánica 1 se consideran de escaso interés botánico, ya que se dedican, fundamentalmente, a cultivos y sufren importantes impactos ambientales, la mayor parte por acciones antrópicas. Las áreas de calidad botánica 2 son de mediano interés botánico, con uso tradicional de cultivos y puntualmente con uso forestal. A pesar de ello poseen interés turístico. Estas áreas deberían utilizarse como zonas de impacto para proteger a las colindantes con un mayor valor botánico 
y ecológico. Las áreas de calidad botánica 3 poseen un interés botánico que puede considerarse relativamente elevado, ya que presentan, con mayor o menor frecuencia, casi todas las formaciones vegetales que potencialmente puede darse en el territorio. Dado el interés turístico y paisajístico se debe procurar un manejo controlado para estos fines. Las áreas de calidad botánica 4 representan las zonas de mayor interés botánico, con las especies y comunidades más singulares. Las comunidades vegetales de estas áreas son muy sensibles a impactos medioambientales, por lo que su manejo debe estar restringido al máximo.

\section{ANEXO 1}

\section{Listado de táxones}

Acaulon fontiquerianum Casas \& Sérgio Amphidium mougeotii (Bruch \& Schimp.) Schimp.

Anacolia menziesii (Turner) Parihar Athalamia spathysii (J.Lindb.) S.Hatt. Atrichum undulatum (Hedw.) P.Beauv. Brachythecium dieckii Röll

Calliergonella cuspidata (Hedw.) Loeske Cephaloziella stellulifera (Taylor ex Spruce) Schiffn.

Crossidium aberrans Holz. \& E.B.Bartr.

Crossidium seriatum H.A.Crum \& Steere

Didymodon sicculus M.J.Cano, Ros, GarcíaZamora \& J.Guerra

Fabronia pusilla Raddi

Funariella curviseta (Schwägr.) Sérgio

Gigaspermum mouretti Corb.

Gongylanthus ericetorum (Raddi) Nees

Homalothecium philippeanum (Spruce) Bruch,

Schimp. \& W.Gümbel

Orthotrichum acuminatum H.Philib.

Orthotrichum macrocephalum Lara, Garilleti \& Mazimpaka

Orthotrichum pumilum Sw.
Orthotrichum tenellum Bruch ex Brid.

Orthotrichum tortidontium Lara, Garilleti \& Mazimpaka

Orthotrichum urnigerum Myrin

Phascum cuynetii Bizot \& R.B.Pierrot ex J.

Guerra, M.N.Jiménez, Ros \& J.S.Carrión

Philonotis fontana (Hedw.) Brid.

Pottia pallida Lindb.

Pterygoneurum sampaianum (A.M.L.Guim.) A.M.L.Guim.

Pyramidula tetragona (Brid.) Brid.

Scapania praetervisa Meyl.

Schistidium pulchrum H.H.Blom

Schistidium singarense (Schiffn.) Laz.

Tortella flavovirens. (Schiffn.) Laz. var. papillosissima Sérgio \& Casas

Tortula israelis Bizot \& F.Bilewsky

Tortula ruraliformis (Besch.) Grout var. subpapillosissima (Bizot \& R.B.Pierrot) W.A.Kramer

Listado de sintáxones

I. Clase MONTIO-CARDAMINETEA BraunBlanquet \& Tüxen 1943

1. Eucladio verticillati-Didymodontetum tophacei Hébrard 1973

2. Rhizomnio punctati-Fissidentetum taxifolii García-Zamora, Ros \& Guerra, 2000

3. Comunidad de Preissia quadrata

II. Clase PLATYHYPNIDIO-FONTINALIETEA ANTIPYRETICAE Philippi 1956

4. Oxyrrhynchietum rusciformis Gams ex Hübschmann 1953

III. Clase CERATODONTO-POLYTRICHETEA PILIFERI Mohan 1978

5. Brachythecietum albicantis Gams ex Neumayr 1971

6. Comunidad de Anacolia menziesii

IV. Clase RACOMITRIETEA HETEROSTICHI Neumayr 1971 
7. Hedwigio-Orthotrichetum rupestris Varo, Zafra \& Mateo 1988

V. Clase DICRANELLETEA HETEROMALLAE Mohan 1978

8. Bartramietum pomiformis Krusenstjerna ex Hübschmann 1967

9. Comunidad de Bartramia ithyphylla

10. Comunidad de Amphidium mougeotii

VI. Clase BARBULETEA UNGUICULATAE Mohan 1978

11. Trichostomo crispuli-Aloinetum aloidis Guerra \& Varo 1981

12. Tortulo subulatae-Syntrichietum ruralis Cano, Ros, Guerra \& García-Zamora 1999

13. Crossidio squamiferi-Aloinetum aloidis Gil 1997

14. Gymnostomo luisieri-Southbyetum nigrellae (Guerra \& Gil 1982) Ros \& Guerra 1987

15. Reboulio hemisphaericaeTargionietum hypophyllae Gil 1997

16. Plagiochasmo rupestris-Athalamietum spathysii García-Zamora, Ros \& Guerra 2000

17. Riccio nigrellae-Oxymitretum paleaceae Ros \& Guerra 1987

18. Fissidento-Funarietum curvisetae During 1981

19. Homalothecio aurei-Pleurochaetetum squarrosae Ros \& Guerra 1987

20. Selaginello denticulataeTimmielletum barbuloidis Cano, Guerra \& Ros 1997

21. Homalothecio aurei-Scleropodietum touretii García-Zamora, Ros \& Guerra 2000

22. Acaulo triquetri-Tortuletum brevissimae Ros \& Guerra 1987

23. Crossidio crassinervis-Aloinetum aloidis Frey, Herrnstadt \& Kürschner 1990

24. Crossidio crassinervis-Tortuletum obtusatae Ros \& Guerra 1987

25. Crossidio crassinervis-Pottietum commutatae Ros \& Guerra 1987
26. Funarietum hygrometricae Engel 1949

VII. Clase GRIMMIETEA ANODONTIS Hadac \& Vondracek in Jezek \& Vondracek 1962

27. Grimmietum orbicularis Allorge ex Demaret 1944

28 Comunidad de Grimmia pulvinata

VIII. Clase NECKERETEA COMPLANATAE Marstaller 1986

29. Homalothecio sericei-Leptodontetum smithii Moya, Ros \& Guerra 1994

IX. Clase FRULLANIO-LEUCODONTETEA SCIUROIDIS Mohan 1978 emend. Marstaller 1985

30. Syntrichietum laevipilae Ochsner 1928

\section{ANEXO 2}

Listado de localidades muestreadas. Details of the studied sites.

1. Cerro del Mojón (Macael). WG6226. 1060 m

2. Cercanías a «Los Polillos» (Tahal). WG6125. $1100 \mathrm{~m}$

3. Barranco de la Golapra (Gérgal). WG3922 $1600 \mathrm{~m}$

4. Cercanías a «La Fuentecilla» (Bacares) WG4223. $1800 \mathrm{~m}$

5. Punto geodésico Tética (Bacares). WG5223 $2080 \mathrm{~m}$

6. Proximidades a la «Casa del Sastre» (Velefique). WG5323. $1700 \mathrm{~m}$

7. Barranco del Fragüero (Tahal). WG6223. $1100 \mathrm{~m}$

8. Fuente de los Borregos (Gérgal). WG3822 $1700 \mathrm{~m}$

9. Barranco del Negro (Gérgal). WG3922. $1650 \mathrm{~m}$

10. Umbría de la «Loma del Cerrillo» (Bacares) WG4022. $1700 \mathrm{~m}$

11. Barranco del Maguillo (Bacares). WG4122. $1700 \mathrm{~m}$

12. Barranco de Julián (Bacares). WG4422. $1650 \mathrm{~m}$

13. Alto de García (Bacares). WG5022. $1800 \mathrm{~m}$

14. Arroyo de los Pastores (Gérgal). WG3321. $1750 \mathrm{~m}$

15. Barranco de Cano (Gérgal). WG3721. $1700 \mathrm{~m}$

16. Cercanías al Calar Alto (Gérgal). WG3920. $2000 \mathrm{~m}$

17. Barranco del Pino (Bacares). WG4221. $1850 \mathrm{~m}$

18. Barranco de Juan Pérez (Bacares). WG4421. 
$1750 \mathrm{~m}$

19. Arroyo Muñoz (Gérgal). WG3520. $1850 \mathrm{~m}$

20. Barranco del Caño (Tahal). WG6220. $1100 \mathrm{~m}$

21. Barranco de la Verruga (Gérgal). WG3619. $1800 \mathrm{~m}$

22. Cercanías a Casa Pasaica. Barranco del Lugar (Velefique). WG5119.1700 m

23. Carretera Cantoria-Uleila del Campo, km 30 de la C-3325 (Benizalón). WG7019. $1100 \mathrm{~m}$

24. Carretera Cantoria-Uleila del Campo, $\mathrm{km} 3 \mathrm{l}$ de la C-3325 (Benizalón). WG7119. $1000 \mathrm{~m}$

25. Carretera Cantoria-Uleila del campo, $\mathrm{km} 33$ de la C-3325 (Benizalón). WG7219. $1000 \mathrm{~m}$

26. «Piedras del Deseo» (Gérgal). WG3118. $2000 \mathrm{~m}$

27. «La Piedra del Sombrerillo» (Gérgal). WG3318. $1900 \mathrm{~m}$

28. Barranco de La Pileta (Gérgal). WG3417. $1600 \mathrm{~m}$

29. Barranco de Lugar (Velefique). WG5218. $1400 \mathrm{~m}$

30. Barranco de Sufli (Senés). WG5818. $1100 \mathrm{~m}$

31. Cercanías a «Las Zorreras» (Tahal). WG6318. $1200 \mathrm{~m}$

32. Carretera Cantoria-Uleila del Campo, km 31,5 de la C-3325 (Benizalón). WG7118. $1050 \mathrm{~m}$

33. Carretera Cantoria-Uleila del Campo, $\mathrm{km} \mathrm{32,5}$ de la C-3325 (Benizalón). WG7218.950 m

34. Cercanías a la venta «La Huertecica» (Lubrín). WG8118. $600 \mathrm{~m}$

35. Alto de Molina. Carretera Senés-Tabernas, km 2 (Senés). WG5917. $1000 \mathrm{~m}$

36. Rambla del Moratón. Carretera Senés-Tahal (Senés). WG6117. $1000 \mathrm{~m}$

37. Carretera Uleila del Campo-Monteagudo, $\mathrm{km}$ 38 de la C-3325 (Uleila del Campo). WG7117. $800 \mathrm{~m}$

38. Loma del Torilillo (Olula de Castro). WG4616. $1350 \mathrm{~m}$

39. Rambla de la Hoya Pardo (Velefique). WG5214. $800 \mathrm{~m}$

40. Barranco Raspajos. Carretera Tallón AltoGérgal km 8 (Gérgal). WG4511. 1050 m

41. Cerro Santiago. Carretera Tallón Alto-Gérgal km 5 (Olula de Castro). WG4511. 1050 m

42. Barranco de Los Segundos (Castro de Filabres). WG5011. $750 \mathrm{~m}$

43. Carretera Senés-Tabernas, km 10 (Benizalón). WG5810. $600 \mathrm{~m}$

44. Cercanías al Cortijo Colorado (Turre). WG9510. $180 \mathrm{~m}$

45. Rambla del Pocico (Turre). WG9610. $170 \mathrm{~m}$

46. Proximidades al Cortijo Grande (Turre). WG9409. $180 \mathrm{~m}$

47. Loma del Colorado (Turre). WG9408. $400 \mathrm{~m}$

48. Cortijo Tremecén (Turre). WG9506. $650 \mathrm{~m}$

49. Carretera Sorbas-Río de Aguas km 4 (Sorbas). WG8105. $400 \mathrm{~m}$

50. Caserío El Puerto (Turre). WG9105. $420 \mathrm{~m}$

51. Caserío El Prado (Turre). WG9004. $550 \mathrm{~m}$
52. Base del Mezquita (Turre). WG9304. $850 \mathrm{~m}$

53. Rambla de Mizala (Sorbas). WG8401. $310 \mathrm{~m}$

54. Barranco Malco (Carboneras). WF9999. $70 \mathrm{~m}$

55. Cañada Blanco (Sorbas). WF8597. $240 \mathrm{~m}$

56. Carretera a Turrillas, a $3 \mathrm{~km}$ de la N-340 (Tabernas). WG6202. $550 \mathrm{~m}$

57. Carretera N-340, km 157 (Tabernas). WG6602. $600 \mathrm{~m}$

58. «Los Encalmados» (Turrillas). WF6799. $750 \mathrm{~m}$

59. Rambla de La Higuerilla (Lucainena de las Torres). WF6899. $700 \mathrm{~m}$

60. Cercanías al cortijo La Cerradilla (Lucainena de las Torres). WF7299. $500 \mathrm{~m}$

61. "Hoya Segura» (Tabernas). WF5496. $700 \mathrm{~m}$

62. «Los Manueles» (Turrillas). WF6496. $1100 \mathrm{~m}$

63. Barranco de la Cueva (Lucainena de las Torres). WF7396. $600 \mathrm{~m}$

64. Rambla de la Sierra (Tabernas). WF6095. $1100 \mathrm{~m}$

65. Barranco del Castro (Almería). WF6195. $1350 \mathrm{~m}$

66. «Las Palomas» (Tabernas). WF6295. $1200 \mathrm{~m}$

67. Proximidades a «El Puntal» 1 (Tabernas) WF5194. $600 \mathrm{~m}$

68. Proximidades a «El Puntal» 2 (Tabernas) WF5294. $800 \mathrm{~m}$

69. Proximidades a «El Puntal» 3 (Tabernas). WF5394. $900 \mathrm{~m}$

70. «Las Tejoneras» (Tabernas). WF5694. $1100 \mathrm{~m}$

71. Pico Sierra Alhamilla (Almería). WF5794. $1300 \mathrm{~m}$

72. Cercanías a las Piedras de Aldana (Tabernas). WF5894. $1200 \mathrm{~m}$

73. Barranco de La Puerca (Almería). WF5994. $1200 \mathrm{~m}$

74. Barranco del Castro (Almería). WF6194. $1200 \mathrm{~m}$

75. Cercanías al pico Colativí (Almería). WF6294. $1340 \mathrm{~m}$

76. Loma Blanca (Turrillas). WF6394. $1000 \mathrm{~m}$

77. Proximidades al barranco de La Puerca (Almería). WF5993. 1100 m

78. Cortijo Frasquito (Níjar). WF6593. $800 \mathrm{~m}$

79. Proximidades a la cortijada de San Ignacio (Níjar). WF7393. $410 \mathrm{~m}$

80. Barranco del Monegue (Pechina). WF5692 $1200 \mathrm{~m}$

81. Rambla Espinaza (Pechina). WF5390. $400 \mathrm{~m}$

82. Rambla Palmilla (Rioja). WF5189. $270 \mathrm{~m}$

83. Barranco de Benítez (Pechina). WF5087. $180 \mathrm{~m}$

84. Cercanías a la cortijada de El Molino de la Balsa Blanca (Níjar). WF8588. $180 \mathrm{~m}$

85. Cerro de la Mata Lobera (Níjar). WF9188. $90 \mathrm{~m}$

86. Falda del pico Jayón (Níjar). WF8587. $370 \mathrm{~m}$

87. Cercanías a la cortijada de los Garrillos. La Serrata (Níjar). WF8286. $190 \mathrm{~m}$

88. Proximidades a la cortijada Balsa Seca (Níjar). WF7384. $100 \mathrm{~m}$

89. Ladera del pico Artichuela (Níjar). WF8582. $160 \mathrm{~m}$

90. «Las Yeseras»(Níjar). WF7480. $200 \mathrm{~m}$ 
91. Cercanías al cortijo de los Tollos (Níjar). WF8780. $80 \mathrm{~m}$

92. Cercanías a la Boca de los Frailes (Níjar). WF7973. $100 \mathrm{~m}$

93. Cortijo Peralta (Níjar). WF8273. $50 \mathrm{~m}$

94. Proximidades al cortijo de la Velasca (Níjar). WF7572. $280 \mathrm{~m}$

95. Rambla de las Higueras (Níjar). WF7672. $200 \mathrm{~m}$

96. Pico Carneros (Níjar). WF7671. $250 \mathrm{~m}$

97. Umbría del cerro de los Frailes (Níjar). WF8271. $400 \mathrm{~m}$

98. Cercanías a la cortijada de El Pozo del Cabo (Almería). WF7070. $10 \mathrm{~m}$

99. Cercanías al cortijo de Doña Angeles (Níjar). WF7870. $40 \mathrm{~m}$

100. Rambla de El Pozo de los Frailes (Níjar). WF7970. $10 \mathrm{~m}$

101. «El Sabinar», falda del pico Rellana (Almería). WF7 $169.100 \mathrm{~m}$

102. Cercanías al cortijo Palmera (Almería). WF7068. $20 \mathrm{~m}$

103. Cercanías a los cortijos del Barranco (Níjar). WF7468. $180 \mathrm{~m}$

104. Camino de la cortijada del Romeral al cortijo del Mónsul (Níjar). WF7668. $120 \mathrm{~m}$

105. Cortijada del Romeral (Níjar). WF7768. $50 \mathrm{~m}$

106. Barranco Pollatos (Níjar). WF7567. $100 \mathrm{~m}$

107. Proximidades al cortijo del Mónsul (Níjar). WF7667. $100 \mathrm{~m}$

108. Barranco del Mónsul. (Níjar). WF7566. $50 \mathrm{~m}$ 109. Campillo del Genovés (Níjar). WF7766. $0 \mathrm{~m}$

110. Fondeadero del Morrón. Playa de los Genoveses (Níjar). WF7866. $0 \mathrm{~m}$

111. Cercanías a casas El Corralete (Almería). WF7 $165.100 \mathrm{~m}$

112. Falda del pico San Francisco (Níjar). WF7265. $100 \mathrm{~m}$

113. Barranco del Negro (Níjar). WF7365. $150 \mathrm{~m}$

114. Barranco del Mónsul (Níjar). WF7565. $100 \mathrm{~m}$

115. Faro de Gata (Níjar). WF7264. $30 \mathrm{~m}$

AGRADECIMIENTOS. Los autores desean expresar su agradecimiento a los revisores anónimos por sus críticas constructivas.

\section{BIBLIOGRAFÍA}

AGUILAR, J., J. FERNÁNDEZ, S. DE HARO, J.A. SÁNCHEZ y J. WALKATE -1988- Hojas de Garrucha y Mojácar. Mapa de suelos. E. 1:100.000. Ministerio de Agricultura, Pesca y Alimentación. ICONA. Universidad de Granada.
Granada

AGUILAR, J., J. FERNÁNDEZ, S. DE HARO, J.A SÁNCHEZ y E. FERNÁNDEZ - 1989- Hoja de Carboneras. Mapa de suelos. E. 1:100.000 Ministerio de Agricultura, Pesca y Alimentación. ICONA. Universidad de Granada. Granada.

AGUILAR, J., J. FERNÁNDEZ, T. RODRÍGUEZ, J.A. SÁNCHEZ y E. FERNÁNDEZ -1990- Hojas de El Cabo de Gata y El Pozo de los Frailes. Mapa de suelos. E. 1:100.000. Ministerio de Agricultura, Pesca y Alimentación. ICONA Universidad de Granada. Granada.

AGUILAR, J., T. RODRÍGUEZ, M. SIMÓN, J. FERNÁNDEZ y J.A. SÁNCHEZ - 1987a- Hoja de Macael. Mapa de suelos. E. 1:100.000 Ministerio de Agricultura, Pesca y Alimentación. ICONA. Universidad de Granada. Granada.

AGUILAR, J., M. SIMÓN, J. FERNÁNDEZ, I. GARCÍA y J.M. MILÁN -1987b-Hoja de Fiñana. Mapa de suelos. E. 1:100.000. Ministerio de Agricultura, Pesca y Alimentación. ICONA. Universidad de Granada Granada

CANO, M.J., R.M. ROS, J. GUERRA \& P. GARCÍAZAMORA - 1999- A new bryophytic community from the mountains of Southern Spain and Morocco: Tortulo subulatae-Syntrichietum ruralis. Cryptogamie, Bryologie 20: 197-202.

CASAS, C. -1991- New checklist of Spanish mosses. Orsis 6: 3-26.

DELGADO, G., R. DELGADO, J. PÁRRAGA, E. GAMIZ, M. SÁNCHEZ, J. MEDINA, J.M MARTÍN y J.L. DÍAZ -199 - Hoja de Vera. Mapa de suelos. E. 1:100.000. Ministerio de Agricultura, Pesca y Alimentación. ICONA. Universidad de Granada. Granada.

GARCÍA-ZAMORA, P., R.M. ROS \& J. GUERRA -1998- Bryophyte flora of the Sierras de Filabres, Cabrera, Alhamilla and Cabo de Gata (Almería, S.E. Spain). J. Bryol. 20: 461-493.

GARCÍA-ZAMORA, P., R.M. ROS y J. GUERRA En prensa- Vegetación briofítica de las sierras de Filabres, Cabrera, Alhamilla y Cabo de Gata (Almería, SE de España). Cryptogamie, Bryologie.

GÉHU, J.M. \& J. GÉHU - 1980-Essai d'objectivation de l'évaluation biologique des milieux naturels. Exemples littoraux. Séminaire de 
Phytosociologie appliquée. Indices biocénotiques. 77-94. Ministère de $1 ф$ Environnement et du Cadre de Vie. Mission des Études et de la Recherche.

GUERRA,J., R. M. ROS, J. DE LAS HERAS, P. GARCÍA-ZAMORA y M. N. JIMÉNEZ MARTÍNEZ - 1989-Estudio de la flora briofítica de la sierra de Alcaraz (Albacete) como base para una evaluación fitobiológica del territorio. I. E. A. Serie 1-Ensayos Históricos y Científicos 39.

MARSTALLER, R. -1993- Synsystematische Übersicht über die Moosgesellschaften Zentraleuropas. Herzogia 9: 513-541.

ORTEGA, E., C. SIERRA, T. RODRÍGUEZ, I. GARCÍA, I. SAURA, A. IRIARTE, J.M. GARZÓN y J. LOZANO-1986- Hoja de Gérgal. Mapa de suelos. E. 1:100.000. Ministerio de Agricultura, Pesca y Alimentación. ICONA. Universidad de Granada. Granada.

PÉREZ PUJALTE, A., C. OYONARTE, J.L. GARCÍA-CHICANO, A. FERNÁNDEZ, R. BURGOS, M.C. QUESADA, J.L. GUIRADO y J.L. DÍAZ -1987- Hoja de Tabernas. Mapa de suelos. E. 1:100.000. Ministerio de Agricultura, Pesca y Alimentación. ICONA. CSIC. Granada.
PÉrez PUjAlte, A., C. OyONARTE, M. TORRES, A. FERNÁNDEZ, R. BURGOS, J.L. GUIRADO, R.M. MENDOZA, J.L.DÍAZ y M. TORRES -1989a- Hoja de Sorbas. Mapa de suelos. E. 1:100.000. Ministerio de Agricultura, Pesca y Alimentación. ICONA. CSIC. Granada. PÉREZ PUJALTE, A., C. OYONARTE, M. TORRES, A. FERNÁNDEZ, R. BURGOS, J.L. GUIRADO, R.M. MENDOZA, B. PÉREZ LOPEZ y J.L. DÍAZ.-1989b- Hoja de Almería. Mapa de suelos. E. 1:100.000. Ministerio de Agricultura, Pesca y Alimentación. ICONA. CSIC. Granada.

Aceptado para su publicación en Octubre de 1999

Dirección de los autores. Departamento de Biología Vegetal (Botánica), Facultad de Biología, Universidad de Murcia, E-30100 Murcia, España. E-mail: rmros@fcu.um.es 